\title{
Predictability and Manageability of Diabetic Complications among Non- Dual Medicare Beneficiaries
}

Ramzi Abujamra', David Randall² ${ }^{\star}$, Stephen T Parente ${ }^{3}$ and Boris Bershadsky ${ }^{4}$

${ }^{1} \mathrm{c} / \mathrm{o}$ American Research and Policy Institute, Washington, DC, USA

${ }^{2}$ American Research and Policy Institute, Suite 200, 1250 Connecticut Ave, NW, Washington, DC, USA

${ }^{3}$ Carlson School, University of Minnesota, Minneapolis, MN, USA

${ }^{4}$ Outcomes Analytics, School of Public Health, University of Minnesota, Minneapolis, MN, USA

\begin{abstract}
Objectives: This study aims to explore factors that influence progression of Diabetes complications among Medicare non-dual beneficiaries. Three Diabetes complications explored are retinopathy, nephropathy and neuropathy. A second objective of this study is to explore the impact that various patient management programs have on reducing the risk of development of Diabetes complications. Three patient management programs are explored, including total cost of treatment (for payer), total patient cost share and physician factors (rural vs. urban and primary care vs. specialist).

Methods: Predictive and descriptive logistic regression models are used with each Diabetes complication as the outcome variable. For predictive models, the risk factors are obtained using stepwise logistics regression. For explanatory models, the risks factors are included with each of the analytical factors in order to risk adjust the impact of the analytical factors on the development of Diabetes complications.

Results: Nephropathy is found to be the most predictive of the three Diabetes complications. For nephropathy, both total cost of treatment and total patient cost share show negative statistical relation with nephropathy development. For physician factors, rural and specialist physicians are found to be associated with lower rate of nephropathy development among beneficiaries.

Conclusions: There is evidence that prediction and management of Diabetes complications can lead to improved outcomes among Medicare beneficiaries. Total cost of treatment, total patient cost share and physician factors (rural vs. urban and primary care vs. specialist) all appear to play a role in improved outcomes in nephropathy development among beneficiaries.
\end{abstract}

\section{Introduction}

Diabetes is one of the leading debilitating chronic conditions, affecting nearly 25 million Americans. It is the $7^{\text {th }}$ leading cause of death among the US population [1]. The total cost of Diabetes in the US is \$245 Billion (2012), with treatment of Diabetes complications costing as high as $\$ 58$ Billion (2007) [2]. Diabetes is more significant among the older population, with 1 in 3 dollars spent on Diabetes treatment among Medicare beneficiaries in the US [1].

This study seeks to explore the development of novel complications among non-dual Medicare beneficiaries who have not previously been observed to have developed any complications. The goal is to explore patient management programs that can alter or at least minimize the impact of these new complications among Medicare beneficiaries. The purpose of this study is to consider two overall goals:

1. Predicting beneficiaries who are likely to develop one of three Diabetes complications in the follow-up year (who have no observed long-term Diabetes complications in the base year)

2. Managing beneficiaries in the base year in order to avoid or minimize the risk of developing a Diabetes complication in the followup year. Three patient management programs are covered: 1) total cost of treatment 2) total patient cost share and 3) physician factors (primary care vs. specialist and urban vs. rural).

Various studies have previously looked at the impact of managing Diabetes patients with impacts on both health outcomes and cost [35]. These studies have typically focused on managing HBA1c levels among patients. There have also been numerous studies that focus on prediction of Diabetes among a cohort. Many of these studies employ sophisticated statistical or machine learning techniques [6,7]. This study hopes to build on this literature with both prediction and patient management programs for those cases that are found to be risky.

\section{Methods and Data}

The data source for this study is Medicare's Limited Data Set (LDS) file for years 2010 and 2011. This data set contains a 5\% sample of Medicare beneficiaries with their full data from the Centers for Medicare and Medicaid Services (CMS) [8]. The file components used include the beneficiary file (or the denominator file) as well as the following five claim utilization files: inpatient (IP), outpatient (OP), carrier (MD), skilled nursing facility (SNF) and home health (HH).

The approach of this study is to consider a cohort of non-dual Medicare beneficiaries who are diagnosed with Diabetes and who do not have any long-term Diabetes complications in the base year. Diabetes beneficiaries are identified as having at least one occurrence of International Classification of Diseases, Ninth Revision (ICD-9) diagnosis codes 250.xx, in both the first and second year [4] for OP

*Corresponding author: David Randall, Executive Director and Resident Scholar American Research and Policy Institute, Suite 200, 1250 Connecticut Ave, NW, Washington, DC, Tel: 202-558-6364; E-mail: info@arapi.org

Received June 24, 2015; Accepted September 11, 2015; Published September 17,2015

Citation: Abujamra R, Randall D, Parente ST, Bershadsky B (2015) Predictability and Manageability of Diabetic Complications among Non-Dual Medicare Beneficiaries. J Health Med Informat 6: 203. doi:10.4172/2157-7420.1000203

Copyright: (c 2015 Abujamra R, et al. This is an open-access article distributed under the terms of the Creative Commons Attribution License, which permits unrestricted use, distribution, and reproduction in any medium, provided the original author and source are credited. 
and MD claim utilization sources, at least two occurrences are required (due to their larger sizes)[9,10]. Beneficiaries are then observed for development of one of three long-term microvascular Diabetes complications in the follow-up year. The three Diabetes complications that are observed are retinopathy, nephropathy and neuropathy. ICD9 code criteria used for these Diabetes complications are: retinopathy (250.5x, 362.0x, 379.23), nephropathy (250.4x, 585.xx, 581.81, 583.81) and neuropathy $(250.6 \mathrm{x}, 357.2 \mathrm{x}, 337.1 \mathrm{x})[4,11]$

There are additional restrictions that are also imposed on the cohort. First, no deceased beneficiaries are included. Also, all claims with negative costs are removed ${ }^{13}$. Beyond these restrictions, additional criteria were to include only beneficiaries who were aged (65+), enrolled in both Medicare Part A and Part B, had 12 months of full enrollment (in both years) and who did not have any managed care enrollment in the study period ${ }^{14}$. The final count of the total beneficiaries that met eligibility for the study was 126,942 beneficiaries.

The variables included in the study, along with a brief description, are shown in the Appendix (Table A1). The variables consist of three categories: socio-demographic, health utilization and comorbidity variables. Comorbidity variables are based on Elixhauser comorbidity factors $[12]^{15}$. These variables are used as the input variables in the model. The outcome variables are the new cases of Diabetes complications that a beneficiary develops (retinopathy, nephropathy and neuropathy). The distribution for those complications, based on the follow-up year, is shown in Table 1.

The first model is the predictive model that identifies the risk factors associated with development of Diabetes complications among Medicare beneficiaries. The second model is the descriptive model that evaluates the impact of the different patient management programs on development of Diabetes complications among beneficiaries. In the descriptive model, three analytical factors are introduced, corresponding with each of the different patient management programs. The first analytical factor is the beneficiary total cost of treatment in the base year (these are the costs incurred by the payer, or CMS). Total costs are obtained as the sum of components from the five of claim utilization sources (IP, OP, MD, SNF and HH). Also, total costs are divided into five equal tier levels (' 0 ' is lowest and ' 4 ' is highest). The second analytical factor is the total patient cost share in the base year-this includes deductible, coinsurance and copayment payments. Similar to total costs, total patient cost share is also obtained as the sum from five claim utilization sources, as well as being divided into five equal tier levels. Finally, physician analytical factors are composed of two parts, primary vs. specialist and urban vs. rural factors. In contrast to the other analytical factors, physician factors are only obtained from the MD claim utilization source (due to data regarding physician utilization being only available from that claim source). However, most beneficiaries appear to have physician utilization and this does not have a significant impact ${ }^{16}$.

The predictive model consists of the variables presented in Table A1. Stepwise logistics regression is used to determine the statistically significant predictive factors for each of the Diabetes complications ${ }^{17}$. The descriptive model consists of the variables used in the predictive

13 Less than $0.1 \%$ of total claims were removed

14 These criteria were obtained from the Research and Data Assistance Center (RESDAC), http://www.resdac.org/

15 Two Elixhauser factors related to Diabetes were removed, leaving a total of 29 Elixhauser factors used in the study.

16 Out of the original 126,942 beneficiaries, 126,950 beneficiaries had physician utilization in the base year.

17 Statistically significant is considered as having $p$-value $<0.05$ in this study.

\begin{tabular}{|c|c|c|c|}
\hline Complication & & Count & Percent \\
\hline Retinopathy & no & 123,615 & $97.38 \%$ \\
\hline Nephropathy & yes & 3,327 & $2.62 \%$ \\
\hline & no & 119,330 & $94.00 \%$ \\
\hline Neuropathy & yes & 7,612 & $6.00 \%$ \\
\hline & no & 120,458 & $94.89 \%$ \\
\hline
\end{tabular}

Table 1: Distribution of the three Diabetes complications in follow-up year

model, along with each of the analytical factors. These variables are used as risk adjusters in the descriptive model, to enable comparison of the risk adjusted impact of the analytical factors on the development of Diabetes complications among beneficiaries. Both the predictive and descriptive models are modeled using logistics regression. The output variables for both models are the three dichotomous Diabetes complications variables. All programming is performed using SAS statistical software, version 9.3 (SAS Institute Inc., Cary, NC).

\section{Results}

\section{Predictability}

The results of the predictive models are shown in Figures 1-3, respectively (corresponding with each of the Diabetes complications). These results show graphs of odds ratios of only those factors that are found to be statistically significant with the Diabetes complications (odds ratios are also arranged in ascending order of significance, where the factor at the bottom of the graph is the most statistically significant).

The results of the predictive models show that the nephropathy model is the most predictive among all of the three complications. This is evidenced with nephropathy having both a higher number of significant factors, as well as tighter intervals for those odds ratios than the other complications ${ }^{18}$. As a result of having the best predictive performance, nephropathy is the only complication presented below with regards to patient management programs.

\section{Manageability}

Both total cost of treatment (for the payer) and patient cost share analytical factors are presented in Table 2 below for nephropathy. These results show the risk adjusted impact of those factors on the development of nephropathy among beneficiaries. For both factors, statistical significance is shown by tier levels (where ' 0 ' is the reference tier). None of the tiers are found to be statistically significant in those results. A different approach was taken that considered alternative risk adjustment than Elixhauser comorbidity factors to see if any of the results would be impacted. Risk adjustment based on CMS Hierarchical Condition Category (HCC) risk score was considered [13] In addition, another approach was used that simply used the sum of the number of Elixhauser comorbidities that a beneficiary has. Both of these approaches yielded negative statistically significant results for both factors on the development of nephropathy among beneficiaries ${ }^{19}$. These results indicate that both a higher treatment cost by the payer, as well as higher total patient cost share, are associated with improved outcomes among beneficiaries with regards to development of nephropathy.

For physician factors, both primary vs. specialty and urban vs. rural factors are presented in Table 3 (where primary ' 1 ' and urban 18 This result is further confirmed with the $\mathrm{C}$-statistic for the predictive models $(0.541,0.639$ and 0.604 for retinopathy, nephropathy and neuropathy, respectively).

19 Three of the four tier levels ('2', '3', and ' 4 ') were found negatively statistically significant for both factors, with both risk adjustment approaches 
Citation: Abujamra R, Randall D, Parente ST, Bershadsky B (2015) Predictability and Manageability of Diabetic Complications among Non-Dual Medicare Beneficiaries. J Health Med Informat 6: 203. doi:10.4172/2157-7420.1000203

\begin{tabular}{|c|c|c|c|c|}
\hline & \multicolumn{2}{|c|}{ Payer (CMS) } & \multicolumn{2}{c|}{ Patient Cost Share } \\
\hline Cost Tiers & Estimate (SE) & $\mathbf{p}$-value & Estimate (SE) & p-value \\
\hline 4 & $.004(.054)$ & 0.9355 & $-.079(.054)$ & 0.1478 \\
\hline 3 & $.056(.045)$ & 0.2176 & $-.0003(.044)$ & 0.9952 \\
\hline 2 & $-.001(.044)$ & 0.9899 & $-.026(.043)$ & 0.5517 \\
\hline 1 & $.076(.042)$ & 0.072 & $.025(.042)$ & 0.554 \\
\hline 0 & 0 &. & 0 &. \\
\hline
\end{tabular}

Table 2: Nephropathy complication outcome for payer and patient cost share, by cost tiers.

\begin{tabular}{|l|l|l|l|}
\hline Provider & & Estimate (SE) & p-value \\
\hline Primary & 0 & $-.010(.030)$ & 0.7315 \\
\hline Urban & 1 & 0 &. \\
\hline & 0 & $-.118(.037)^{\star}$ & 0.0013 \\
\hline & 1 & 0 &. \\
\hline
\end{tabular}

Table 3: Nephropathy complication outcome by provider type (primary vs specialty, urban vs rural)

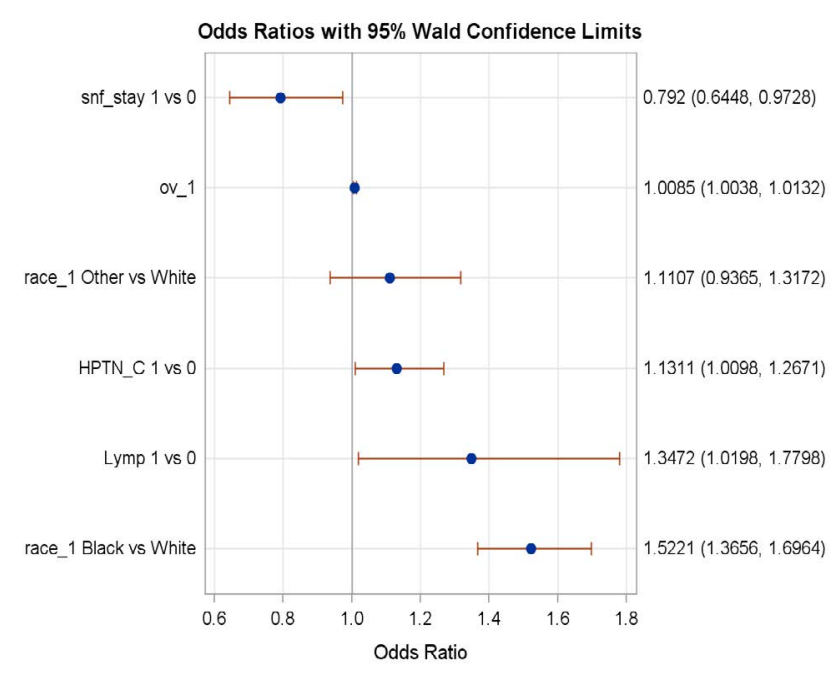

Figure 1: Odds ratio results for retinopathy Diabetes complication predictive model.

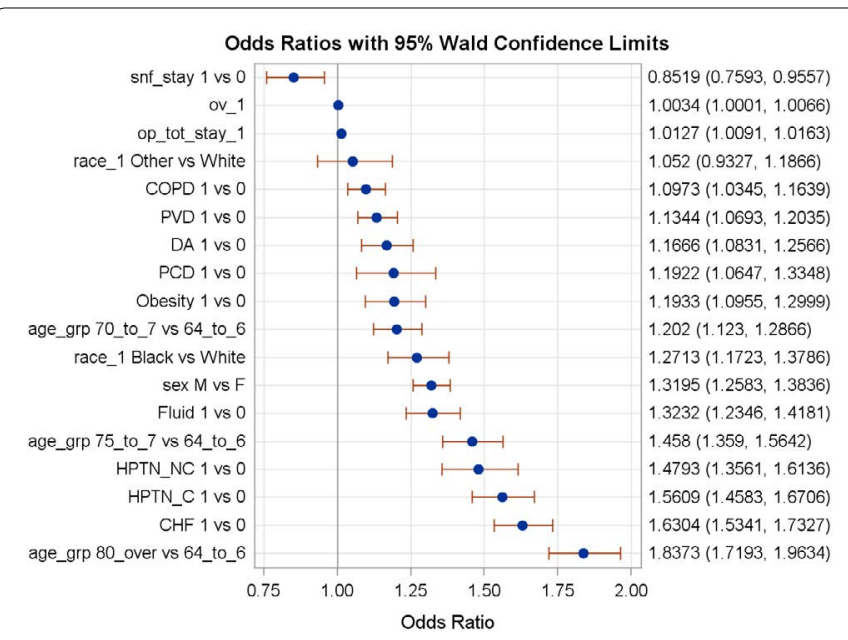

Figure 2: Odds ratio results for nephropathy Diabetes complication predictive model.

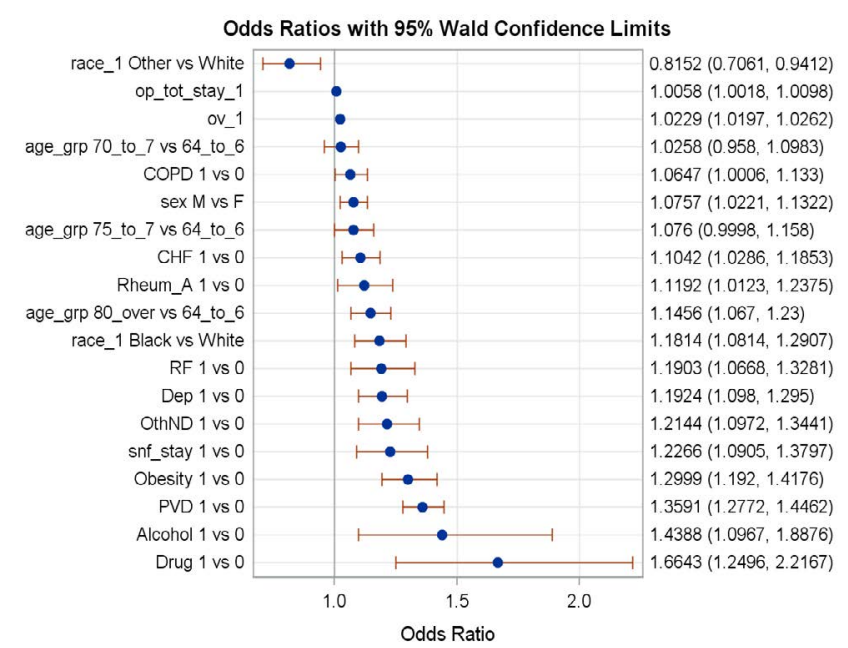

Figure 3: Odds ratio results for neuropathy Diabetes complication predictive model.

' 1 ' are the reference levels, respectively). Only urban vs. rural factor is statistically significant. The results also indicate that rural providers have better outcomes on beneficiaries with regards to development of nephropathy. However, using the other risk adjustment approaches, primary vs. specialist factor is also found to be statistically significant showing evidence that specialists have better outcomes than primary care physicians on development of nephropathy among beneficiaries.

\section{Conclusion and Implications}

This study identifies the risk factors for each of three Diabetes complications among non-dual Medicare beneficiaries. We find that nephropathy was the most predictable of the three Diabetes complications considered. Furthermore, for nephropathy, we find that total amount invested, both by the payer (CMS) and the patient (in the form of cost share), can lead to lower risk of developing nephropathy among beneficiaries with no previous Diabetes complications. We also note that higher investment by both payer and patient is associated with improved outcomes for nephropathy. This result underscores the importance of the increasing role of the patient in paying for care in high deductible health plans [14]. For physician factors, both rural and specialist physicians appeared to have an improved outcome on beneficiaries with regards to developing nephropathy. These results highlight the importance of both predicting and managing Diabetes complications among beneficiaries. Future studies can consider more sophisticated prediction models for the complications. In addition, alternative patient management programs could be explored that lead to better management of those beneficiaries identified as risky for a given complication.

\section{References}

1. Centers for Disease Control and Prevention, National Diabetes Fact Sheet: United States (2011) US Department of Health and Human Services: Atlanta.

2. American Diabetes Association, Economic costs of diabetes in the US in 2007 (2008) Diabetes Care 31: 596-615.

3. Bertoni AG, Hundley WG, Massing MW, Bonds DE, Burke GL, et al. (2004) Heart failure prevalence, incidence, and mortality in the elderly with diabetes. Diabetes Care 27: 699-703.

4. Menzin J, Korn JR, Cohen J, Lobo F, Zhang B, et al. (2010) Relationship between glycemic control and diabetes-related hospital costs in patients with type 1 or type 2 diabetes mellitus. Journal of Managed Care Pharmacy 16: 264-275. 
Citation: Abujamra R, Randall D, Parente ST, Bershadsky B (2015) Predictability and Manageability of Diabetic Complications among Non-Dual Medicare Beneficiaries. J Health Med Informat 6: 203. doi:10.4172/2157-7420.1000203

Page 4 of 4

5. Wagner EH, Sandhu N, Newton KM, McCulloch DK, Ramsey SD, et al. (2001) Effect of improved glycemic control on health care costs and utilization. Jama 285:182-189.

6. Davis DA, Chawla N, Blumm N (2008) Predicting individual disease risk based on medical history. In Proceedings of the $17^{\text {th }}$ ACM conference on Information and knowledge management.

7. Moturu ST, Johnson WG, Liu H (2010) Predictive risk modelling for forecasting high-cost patients: a real-world application using Medicaid data. International Journal of Biomedical Engineering and Technology 3: 114-132.

8. Parente ST, Weiner JP, Garnick DW, Richards TM, Fowles J et al. (1995) Developing a quality improvement database using health insurance data: a guided tour with application to Medicare's National Claims History file. Am J Med Qual 10: 162-176.

9. Hebert PL, Geiss LS, Tierney EF, Engelgau MM, Yawn BP, et al, (1999) Identifying persons with diabetes using Medicare claims data. Am J Med Qual 14: $270-277$
10. Rector TS, Wickstrom SL, Shah M, Thomas GN, Rheault P, et al. (2004) Specificity and Sensitivity of Claims $\square$ Based Algorithms for Identifying Members of Medicare+ Choice Health Plans That Have Chronic Medical Conditions. Health serv res 39: 1839-1858.

11. Selby JV, Thomas R, Dany Z, Chris JC (1997) Excess costs of medical care for patients with diabetes in a managed care population. Diabetes care 20 1396-1402.

12. Quan H, Sundararajan V, Halfon P, Fong A, Burnand B, et al. (2005) Coding algorithms for defining comorbidities in ICD-9-CM and ICD-10 administrative data. Medical care 43: 1130-1139.

13. Pope GC, Kautter J, Ellis RP, Ash AS, Ayanian JZ, et al. (2004) Risk adjustment of Medicare capitation payments using the CMS-HCC model. Health Care Financ Rev 25: 119-141.

14. Parente ST and Randall D (2012) "Impact of High Deductible Health Plans: Point-Counterpoint." Debates on U.S. Health Care Reform 525-535. 